\title{
Discretized optimal control approach for dynamic Multi-Agent decentralized coverage
}

\author{
Minh Tri Nguyen, Luis Rodrigues, Cristina Stoica Maniu, Sorin Olaru
}

\begin{abstract}
This paper presents a novel discrete-time decentralized control law for the Voronoi-based self-deployment of a Multi-Agent dynamical system. The basic control objective is to let the agents deploy into a bounded convex polyhedral region and maximize the coverage quality by computing locally the control action for each agent. The Voronoi tessellation algorithm is employed to partition dynamically the deployed region and to allocate each agent to a corresponding bounded functioning zone at each time instant. The control synthesis is then locally computed based on an optimal formulation framework related to the Lloyd's algorithm but according to the discrete-time agent's dynamics equation. The performance of the discretized optimal solution will be demonstrated via an illustrative example.
\end{abstract}

Index Terms-Multi-agent dynamical systems, Voronoi partition, discrete-time systems.

\section{INTRODUCTION}

The attention for Multi-Agent Dynamical Systems (MAS) has been raised in recent years due to their various applications ranging from research operations (meteorological monitoring, geological exploration etc.) to domestic utilities (mini mobile robots, manufacturing etc.). Among the most well-known MAS applications the deployment task is widely considered leading to distributing a group of independent but cooperative mobile agents (vehicle, robots, etc.) over a predetermined region (e.g. [1], [2]). The ultimate goal of such applications is to maximize the coverage quality subject to selected constraints, e.g. energy-efficient consumption [3], [2], environmental disturbance [4], [5] or obstacle avoidance [6], [7]. This goal is translated in terms of driving the agents into a static partition considered as optimal coverage with respect to a given criterion. There are various works mentioned in the control literature employing dynamic Voronoi partition [8] as a conventional mathematical tool to approach a stationary configuration over a given bounded region. Many recent research works focus on driving the MAS into a Centroidal Voronoi Configuration (CVC) in which the position of each agent coincides with the centroid (center of mass) of its associated Voronoi cell ${ }^{1}$ [9], [3], [10], [11]. The

M.T. Nguyen, C. Stoica Maniu and S. Olaru are with Laboratoire des Signaux et Systèmes (L2S, UMR CNRS 8506), CentraleSupélecCNRS-Univ. Paris-Sud, Université Paris Saclay, 3 rue Joliot Curie, F91192, Gif-sur-Yvette cedex, France (e-mail: \{minhtri.nguyen; cristina.stoica; sorin.olaru\}esupelec.fr). L. Rodrigues is with the Department of Electrical and Computer Engineering, Concordia University, Montreal, QC H3G 2W1, Canada (e-mail: luisrodeencs.concordia.ca).

${ }^{1}$ A Voronoi partition can be obtained in a distributed manner by considering that each agent construction relies only on its local state and the information of its closest neighbors, to construct its own Voronoi cell.
Lloyd's algorithm (see [12]) is widely used as a conventional tool to approximately compute a CVC by moving the agents toward their corresponding centers of mass.

In [9], the authors propose a generalized power-weighted Voronoi partition and modify the Lloyd's algorithm to solve the power constrained deployment. The results in [3] aim to reduce the step size in the Lloyd's algorithm. Other results [13] use Mixed-Integer Programming to minimize the energy consumption. In the notable recent work [10], the coverage task is formulated as an optimal control problem, which considers energy constraints in the control law computation. The authors show that the Lloyd's algorithm-based local control may waste too much energy and they propose an improved energy-efficient optimal control. The main results are developed for continuous-time systems.

The main contribution of the current paper is to extend the decentralized optimal control in [10] for discrete-time systems. The main novelty is to use the prediction of the dynamic Voronoi partition in order to enhance the coverage quality by driving the Multi-Agent system to a CVC. This is chosen as a conventional optimal coverage of the selfdeployment problem.

This paper is structured as follows. Section II presents some useful preliminaries. The main results of [10] are briefly recalled in Section III. The proposed discrete-time solution is detailed in Section IV, followed by the stability proof for the entire MAS in Section V. Concluding remarks and perspectives are mentioned in the last section.

Notation: We use $\|x\|=\sqrt{x^{\top} x}$ to denote the Euclidean norm of the given vector $x$. We denote by $\mathcal{N}_{i}$ the set containing the neighbor's indices of the $i^{t h}$ agent.

\section{PROBLEM FORMULATION}

\section{A. System description}

Consider the Multi-Agent system (denoted by $\boldsymbol{\Sigma}$ ) composed of $N$ mobile agents. The indices set is $\mathcal{N}=$ $\{1, \ldots, N\}$. Each agent has its own dynamics

$$
\dot{x}_{i}=u_{i} \text {, with } i \in \mathcal{N}
$$

which has the discrete-time zero-order-hold form

$$
x_{i}(k+1)=x_{i}(k)+T_{s} u_{i}(k), \text { with } i \in \mathcal{N}
$$

where $x_{i} \in \mathbb{R}^{n}$ is the state-space vector, $u_{i} \in \mathbb{R}^{n}$ is the input vector ${ }^{2}$ and $T_{s}$ is the sampling time. We use $x=$ $\left[x_{1}^{\top} \ldots x_{N}^{\top}\right]^{\top}$ and $u=\left[u_{1}^{\top} \ldots u_{N}^{\top}\right]^{\top}$ to denote respectively the collective state and input of the system $\boldsymbol{\Sigma}$.

\footnotetext{
${ }^{2}$ The dimension choice of $u_{i}$ follows the chosen dynamics (1). The results can be extended for $u_{i} \in \mathbb{R}^{m}$, with $m \neq n$.
} 


\section{B. Constraints on the agents' environment}

Assume that the common working space $\mathcal{X} \subset \mathbb{R}^{n}$ is convex and bounded, represented by a polytope. A partition $\mathbb{V}\left(x_{1}, \ldots, x_{N}\right)$ of $\mathcal{X}$ is

$$
\mathcal{X}=\bigcup_{i=1}^{N} \mathbb{V}_{i}, \quad \mathbb{V}_{i} \cap \mathbb{V}_{j}=\emptyset, \quad \forall i, j \in \mathcal{N}
$$

A natural mathematical definition of such a decomposition is provided by the Voronoi partition, which characterizes the neighborhood $\mathbb{V}_{i}\left(x_{i}\right)$ as

$$
\mathbb{V}_{i}=\left\{x \in \mathcal{X} \mid\left\|x_{i}-x\right\| \leq\left\|x_{j}-x\right\|, \forall j \neq i\right\}
$$

It is worth to be mentioned that each set $\mathbb{V}_{i}$ is a polytope as a consequence of the boundedness of $\mathcal{X}$ and the structure of the constraints in (4). Using the available state measurement of the Multi-Agent system $\boldsymbol{\Sigma}$ at the time instant $k$, the geometric formulation (3) leads to a time-varying partition.

\section{Coverage control}

The ultimate goal of the deployment is to maximize the coverage. The density function ${ }^{3} \phi: \mathcal{X} \rightarrow \mathbb{R}_{+}$denotes the priority of coverage at a point $q \in \mathcal{X}$. A candidate Lyapunov function $V(x)$ (see [11], [14]) is defined as

$$
V(x)=\sum_{i=1}^{N} p_{i} \int_{\mathbb{V}_{i}}\left\|x_{i}-q\right\|^{2} \phi(q) d q
$$

with the positive scalar $p_{i}$ denoting a weighting coefficient for $V(x)$. The optimal (maximized) coverage is achieved if $V(x)$ reaches its minimum.

Its local minimum points are obtained by solving $\frac{\partial V}{\partial x_{i}}=0$ with the partial derivative of $V(x)$ with respect to $x_{i}$ being

$$
\begin{aligned}
& \frac{\partial V}{\partial x_{i}}=2 p_{i} \int_{\mathbb{V}_{i}}\left(x_{i}-q\right)^{\top} \phi(q) d q \\
& =2 p_{i}\left(\int_{\mathbb{V}_{i}} \phi(q) d q\right)\left(x_{i}-\frac{\int_{\mathbb{V}_{i}} q \phi(q) d q}{\int_{\mathbb{V}_{i}} \phi(q) d q}\right)^{\top} \\
& =2 p_{i} M_{\mathbb{V}_{i}}\left(x_{i}-C M_{\mathbb{V}_{i}}\right)^{\top}
\end{aligned}
$$

where the mass $M_{\mathbb{V}_{i}}$ and the center of mass $C M_{\mathbb{V}_{i}}$ of the Voronoi cell $\mathbb{V}_{i}$ are respectively defined as in [10]

$$
\begin{gathered}
M_{\mathbb{V}_{i}}=\int_{\mathbb{V}_{i}} \phi(q) d q \\
C M_{\mathbb{V}_{i}}=\frac{\int_{\mathbb{V}_{i}} q \phi(q) d q}{\int_{\mathbb{V}_{i}} \phi(q) d q}
\end{gathered}
$$

Solving $\frac{\partial V}{\partial x_{i}}=0, \forall i \in \mathcal{N}$ leads to an optimal configuration where $x_{i}=C M_{\mathbb{V}_{i}}$. Such optimal configuration is called Centroidal Voronoi Configuration (CVC). Using LaSalle's invariance principle (see [15]), it can be proved that the agent's local control $u_{i}=k_{i}\left(x_{i}-C M_{\mathbb{V}_{i}}\right)$, with $k_{i}<0$ can lead to the convergence of the entire MAS to a CVC.

The results obtained for the continuous-time MAS will be recalled in the next section.

\footnotetext{
${ }^{3}$ The function $\phi$ is continuously differentiable over $\mathcal{X}$.
}

\section{CONTINUOUS-TIME DECENTRALIZED OPTIMAL CONTROL}

This section recalls the main results given in [10], applied for a MAS whose agents dynamics are characterized by the continuous-time equations (1). The decentralized control is obtained locally by solving the following optimization control problem

$$
\inf _{u_{i}, i \in \mathcal{N}} \int_{0}^{\infty} L(x, u) d \tau \text { s.t.: } \dot{x}_{i}=u_{i}
$$

where $L(x, u)=\sum_{i=1}^{N}\left(s_{i}\left\|\int_{\mathbb{V}_{i}}\left(x_{i}-q\right) \phi(q) d q\right\|^{2}+r_{i}\left\|u_{i}\right\|^{2}\right)$ denotes the running cost. The scalars $s_{i}>0, r_{i}>0$ represent the weighting coefficients. The Hamilton-Jacobi-Bellman (HJB) equation of (9) with the dynamics (1) is

$$
\inf _{u_{i}, i \in \mathcal{N}} H\left(L(x, u), \frac{\partial V}{\partial x}\right)=0
$$

with the Hamiltonian

$$
\begin{aligned}
H & =L(x, u)+\frac{\partial V}{\partial x} \dot{x} \\
& =\sum_{i=1}^{N}\left(s_{i}\left\|\int_{\mathbb{V}_{i}}\left(x_{i}-q\right) \phi(q) d q\right\|^{2}+r_{i}\left\|u_{i}\right\|^{2}+\frac{\partial V}{\partial x_{i}} \dot{x}_{i}\right)
\end{aligned}
$$

associated with a value function $V=V(x)$

$$
V(x)=\sum_{i=1}^{N} \sqrt{s_{i} r_{i}} \int_{\mathbb{V}_{i}}\left\|x_{i}-q\right\|^{2} \phi(q) d q
$$

with $s_{i} r_{i}=s_{j} r_{j}=p^{2}, \forall i, j \in \mathcal{N}$ and $p$ denoting a positive scalar constant. By solving $\frac{\partial H}{\partial u_{i}}=0$, we obtain the continuous-time decentralized optimal control (CDOC)

$$
u_{i}=-\sqrt{\frac{s_{i}}{r_{i}}} \int_{\mathbb{V}_{i}}\left(x_{i}-q\right) \phi(q) d q, \quad i \in \mathcal{N}
$$

Furthermore, the solution (12) satisfies LaSalle's invariance principle and thus ensures the Lyapunov convergence of the MAS into a CVC by means of $\dot{V}=-L \leq 0$.

\section{DisCRETE-TIME DECENTRALIZED OPTIMAL CONTROL}

This section proposes an optimal control for the discretetime system's dynamics (2), based on the approach developed in [10]. For brevity, in the sequel we use $\mathbb{V}_{i}^{+}=\mathbb{V}_{i}\left(x_{i}(k+1)\right)$ to denote the Voronoi cell of $x_{i}(k+1)$, i.e. the $i$-th agent at time $k+1$. The mass and center of mass of the Voronoi cell $\mathbb{V}_{i}^{+}$are respectively $M_{\mathbb{V}_{i}^{+}}$and $C M_{\mathbb{V}_{i}^{+}}$.

Transposing the previous work [10] to the discrete-time dynamics (2) yields the following optimization problem

$$
\begin{aligned}
& \min _{u_{i}, i \in \mathcal{N}} \sum_{k=0}^{\infty} L(x(k), u(k)) \\
& \text { s.t: } x_{i}(k+1)=x_{i}(k)+T_{s} u_{i}(k)
\end{aligned}
$$

with $L(x(k), u(k))=\sum_{i=1}^{N}\left(s_{i}\left\|\int_{\mathbb{V}_{i}}\left(x_{i}(k)-q\right) \phi(q) d q\right\|^{2}+\right.$ $\left.r_{i}\left\|u_{i}(k)\right\|^{2}\right)$ indicating the running cost. 
Theorem 1. A discrete-time decentralized stabilizing suboptimal control of the problem (13) is

$$
u_{i}(k)=-\frac{p_{i}}{r_{i}+p_{i} T_{s} M_{\mathbb{V}_{i}^{+}}} \int_{\mathbb{V}_{i}^{+}}\left(x_{i}(k)-q\right) \phi(q) d q
$$

by considering the cost-to-go function

$$
V(x(k))=\sum_{i=1}^{N} p_{i} \int_{\mathbb{V}_{i}}\left\|x_{i}(k)-q\right\|^{2} \phi(q) d q
$$

with $p_{i}=\sqrt{s_{i} r_{i}}$ and $p_{i}=p_{j}=p>0, \forall i, j \in \mathcal{N}$.

Proof. The discrete-time Hamiltonian (see [16]) of (13) is

$$
H(k)=L(x(k), u(k))+\frac{\Delta V(k)}{T_{s}}
$$

with $\Delta V(k)=V(x(k+1))-V(x(k))$. By using (15), we can express $\Delta V(k)$ as $\Delta V(k)=\sum_{i=1}^{N} p \int_{\mathbb{V}_{i}^{+}}\left\|x_{i}(k+1)-q\right\|^{2} \phi(q) d q-$ $\sum_{i=1}^{N} p \int_{\mathbb{V}_{i}}\left\|x_{i}(k)-q\right\|^{2} \phi(q) d q$. Using the expressions (6)-(8), this can be rewritten in terms of the mass and the center of mass such as $\Delta V(k)=$ $\sum_{i=1}^{N} p\left(\left\|x_{i}(k+1)\right\|^{2} M_{\mathbb{V}_{i}^{+}}+\int_{\mathbb{V}_{i}^{+}}\|q\|^{2} \phi(q) d q-\right.$ $\left.2 x_{i}^{\top}(k+1) M_{\mathbb{V}_{i}^{+}} C M_{\mathbb{V}_{i}^{+}}\right)-\sum_{i=1}^{N} p\left(\left\|x_{i}(k)\right\|^{2} M_{\mathbb{V}_{i}}-\right.$ $\left.2 x_{i}^{\top}(k) M_{\mathbb{V}_{i}} C M_{\mathbb{V}_{i}}+\int_{\mathbb{V}_{i}}\|q\|^{2} \phi(q) d q\right)$. By following the definition (3)-(4), we have $\sum_{i=1}^{N} \int_{\mathbb{V}_{i}}\|q\|^{2} \phi(q) d q=$ $\int_{\bigcup_{i=1}^{N} \mathbb{V}_{i}}\|q\|^{2} \phi(q) d q=\int_{\mathcal{X}}\|q\|^{2} \phi(q) d q$ and this leads to $\sum_{i=1}^{N} \int_{\mathbb{V}_{i}^{+}}\|q\|^{2} \phi(q) d q-\sum_{i=1}^{N} \int_{\mathbb{V}_{i}}\|q\|^{2} \phi(q) d q=0$. This allows us to rewrite $\Delta V(k)$ as follows

$$
\begin{aligned}
& \Delta V(k)=\sum_{i=1}^{N} p\left(\left\|x_{i}(k+1)\right\|^{2}-2 x_{i}^{\top}(k+1) C M_{\mathbb{V}_{i}^{+}}\right) M_{\mathbb{V}_{i}^{+}} \\
& -\sum_{i=1}^{N} p\left(\left\|x_{i}(k)\right\|^{2} M_{\mathbb{V}_{i}}-2 x_{i}^{\top}(k) M_{\mathbb{V}_{i}} C M_{\mathbb{V}_{i}}\right)
\end{aligned}
$$

Differentiating $H(k)$ with respect to $u_{i}(k)$, we obtain

$$
\frac{\partial H(k)}{\partial u_{i}(k)}=2 r_{i} u_{i}^{\top}(k)+\frac{1}{T_{s}} \frac{\partial \Delta V(k)}{\partial u_{i}(k)}
$$

where the partial derivative of $\Delta V(k)$ with respect to $u_{i}(k)$ can be obtained by differentiating (17) by $u_{i}(k)$ (using the dynamics (2)), i.e.

$$
\frac{\partial \Delta V(k)}{\partial u_{i}(k)}=2 p T_{s} M_{\mathbb{V}_{i}^{+}}\left(x_{i}(k+1)-C M_{\mathbb{V}_{i}^{+}}\right)^{\top}+T_{s} \theta_{i}
$$

where

$\theta_{i}=\frac{p}{T_{s}}\left(\left\|x_{i}(k+1)\right\|^{2} \frac{\partial M_{\mathbb{V}_{i}^{+}}}{\partial u_{i}(k)}-2 x_{i}^{\top}(k+\right.$

1) $\left.\frac{\partial\left(M_{\mathbb{V}_{i}^{+}} C M_{\mathbb{V}_{i}^{+}}\right)}{\partial u_{i}(k)}\right)+\frac{1}{T_{s}} \sum_{j \in \mathcal{N}_{i}} p\left(\left\|x_{j}(k+1)\right\|^{2} \frac{\partial M_{\mathbb{V}_{j}^{+}}}{\partial u_{i}(k)}-\right.$

$\left.2 x_{j}^{\top}(k+1) \frac{\partial\left(M_{\mathbb{V}_{j}^{+}} C M_{\mathbb{V}_{j}^{+}}\right)}{\partial u_{i}(k)}\right)$

collects all partial derivatives of the mass and center of mass with respect to $u_{i}(k)$.

Replacing (19) in (18), we get $\frac{\partial H(k)}{\partial u_{i}(k)}=2 r_{i} u_{i}^{\top}(k)+$ $2 p x_{i}^{\top}(k+1) M_{\mathbb{V}_{i}^{+}}-2 p M_{\mathbb{V}_{i}^{+}} C M_{\mathbb{V}_{i}^{+}}^{\top}+\theta_{i}$. Using the discretetime dynamics (2) and regrouping the terms related to the control $u_{i}(k)$, the following expression is obtained

$$
\begin{aligned}
\frac{\partial H(k)}{\partial u_{i}(k)}= & 2\left(r_{i}+p T_{s} M_{\mathbb{V}_{i}^{+}}\right) u_{i}^{\top}(k) \\
& +2 p M_{\mathbb{V}_{i}^{+}}\left(x_{i}(k)-C M_{\mathbb{V}_{i}^{+}}\right)^{\top}+\theta_{i}
\end{aligned}
$$

A suboptimal solution $u_{i}(k)$ can be obtained by solving $\left(r_{i}+p T_{s} M_{\mathbb{V}_{i}^{+}}\right) u_{i}^{\top}(k)+p M_{\mathbb{V}_{i}^{+}}\left(x_{i}(k)-C M_{\mathbb{V}_{i}^{+}}\right)^{\top}=0$, i.e.

$$
u_{i}(k)=-\frac{p M_{\mathbb{V}_{i}^{+}}}{r_{i}+p T_{s} M_{\mathbb{V}_{i}^{+}}}\left(x_{i}(k)-C M_{\mathbb{V}_{i}^{+}}\right)
$$

which is also the control solution (14) and the proof of the main claim is completed.

With respect to the local stability of the $i^{t h}$ agent in case of static Voronoi partition, by replacing (14) in (2), the closedloop dynamics becomes

$x_{i}(k+1)=\frac{r_{i}}{r_{i}+p_{i} T_{s} M_{\mathbb{V}_{i}^{+}}} x_{i}(k)+\frac{p_{i} T_{s} M_{\mathbb{V}_{i}^{+}}}{r_{i}+p_{i} T_{s} M_{\mathbb{V}_{i}^{+}}} C M_{\mathbb{V}_{i}^{+}}$.

Obviously,

$$
\frac{r_{i}}{r_{i}+p_{i} T_{s} M_{\mathbb{V}_{i}^{+}}} \leq 1
$$

because $r_{i}, p_{i}, T_{s}, M_{\mathbb{V}_{i}^{+}}>0$.

Remark 1. In Theorem 1, the general case $p_{i}=p_{j}=p$ was considered. Notice that we can choose $p=1$ in order to keep the consistency with the results proposed in [10].

Remark 2. The computation of the control (14) requires the predicted mass $M_{\mathbb{V}_{i}^{+}}$and the predicted center of mass $C M_{\mathbb{V}_{i}^{+}}$of the Voronoi cell $\mathbb{V}_{i}^{+}$. This is considered as the main difference by comparison with the continuous-time case.

Lemma 1. The suboptimal solution (21) can be considered as a discrete-time approximation of the continuous-time decentralized optimal control of the problem (13) because the terms $\theta_{i}$ satisfy

$$
\sum_{i=1}^{N} \int \theta_{i} d u_{i}(k)=0
$$

Proof. Using the control solution (21) translates the expression (20) into $\frac{\partial H(k)}{\partial u_{i}(k)}=\theta_{i}$. According to the definition of 
total derivative of multi-variable function, we can integrate $\frac{\partial H(k)}{\partial u_{i}(k)}$ with respect to all $u_{i}(k)$ and thus we obtain

$$
H(k)=\sum_{i=1}^{N} \int \theta_{i} d u_{i}(k)
$$

Consider the mass conservation law (see [14]), i.e.

$$
\begin{aligned}
& \frac{\partial}{\partial x_{i}} \int_{\mathbb{V}_{i}}\left\|x_{i}-q\right\|^{2} \phi(q) d q=\int_{\mathbb{V}_{i}} \frac{\partial}{\partial x_{i}}\left\|x_{i}-q\right\|^{2} \phi(q) d q \\
& +\int_{\partial \mathbb{V}_{i}}\left\|x_{i}-\gamma\right\|^{2} \phi(\gamma) n^{\top}(\gamma) \frac{\partial \gamma}{\partial x_{i}} d \gamma
\end{aligned}
$$

where $\partial \mathbb{V}_{i}$ is the boundary of the set $\mathbb{V}_{i}$, i.e. $\partial \mathbb{V}_{i}=$ $\bigcup_{\forall j \in \mathcal{N}_{i}}\left(\mathbb{V}_{i} \cap \mathbb{V}_{j}\right)$. Here, $n(\gamma)$ is the unit outward normal to $\partial \mathbb{V}_{i}$ which is parameterized by the scalar $\gamma$. Similar to (24), for the function (15), we obtain $\frac{\partial V(x)}{\partial x_{i}}=p \frac{\partial}{\partial x_{i}} \int_{\mathbb{V}_{i}}\left\|x_{i}-q\right\|^{2} \phi(q) d q$ and derive $\frac{\partial V(x)}{\partial x_{i}}=$ $2 p \int_{\mathbb{V}_{i}}\left(x_{i}-q\right)^{\top} \phi(q) d q+p \int_{\partial \mathbb{V}_{i}}\left\|x_{i}-\gamma\right\|^{2} \phi(\gamma) n^{\top}(\gamma) \frac{\partial \gamma}{\partial x_{i}} d \gamma$

Based on (6), the discrete-time form at the time instant $k+1$ is further obtained

$$
\begin{array}{r}
\frac{\partial V(x(k+1))}{\partial x_{i}(k+1)}=2 p\left(M_{\mathbb{V}_{i}^{+}} x_{i}(k+1)-M_{\mathbb{V}_{i}^{+}} C M_{\mathbb{V}_{i}^{+}}\right)^{\top} \\
+p \int_{\partial \mathbb{V}_{i}^{+}}\left\|x_{i}(k+1)-\gamma\right\|^{2} \phi(\gamma) n^{\top}(\gamma) \frac{\partial \gamma}{\partial x_{i}} d \gamma
\end{array}
$$

Using (2), we have additionally

$$
\frac{\partial \Delta V}{\partial u_{i}(k)}=\frac{\partial V(x(k+1))}{\partial x_{i}(k+1)} \frac{\partial x_{i}(k+1)}{\partial u_{i}(k)}=\frac{\partial V(x(k+1))}{\partial x_{i}(k+1)} T_{s}
$$

By substituting (25) in (26), we obtain

$$
\begin{aligned}
\frac{\partial \Delta V}{\partial u_{i}(k)}= & 2 p T_{s}\left(M_{\mathbb{V}_{i}^{+}} x_{i}(k+1)-M_{\mathbb{V}_{i}^{+}} C M_{\mathbb{V}_{i}^{+}}\right)^{\top} \\
& +T_{s} p \int_{\partial \mathbb{V}_{i}^{+}}\left\|x_{i}(k+1)-\gamma\right\|^{2} \phi(\gamma) n^{\top}(\gamma) \frac{\partial \gamma}{\partial x_{i}} d \gamma
\end{aligned}
$$

Subsequently, from (19) and (27), we get another equation characterizing $\theta_{i}$

$$
\theta_{i}=p \int_{\partial \mathbb{V}_{i}^{+}}\left\|x_{i}(k+1)-\gamma\right\|^{2} \phi(\gamma) n^{\top}(\gamma) \frac{\partial \gamma}{\partial x_{i}} d \gamma
$$

Furthermore, the authors of [10] proved that

$$
\begin{aligned}
& \int_{\partial \mathbb{V}_{i}}\left\|x_{i}-\gamma\right\|^{2} \phi(\gamma) n^{\top}(\gamma) \frac{\partial \gamma}{\partial x_{i}} d \gamma \\
& =\sum_{j \in \mathcal{N}_{i}} \int_{\mathbb{V}_{i} \cap \mathbb{V}_{j}}\left\|x_{i}-\gamma_{i j}\right\|^{2} \phi\left(\gamma_{i j}\right) n^{\top}\left(\gamma_{i j}\right) \frac{\partial \gamma_{i j}}{\partial x_{i}} d \gamma_{i j}
\end{aligned}
$$

which represents the mass variation through the boundary of $\mathbb{V}_{i}$ as a collection of mass flow through each facet $\mathbb{V}_{i} \cap \mathbb{V}_{j}$ defining $\partial \mathbb{V}_{i}$. Therefore, we obtain

$\theta_{i}=p \sum_{j \in \mathcal{N}_{i}} \int_{\mathbb{V}_{i}^{+} \cap \mathbb{V}_{j}^{+}}\left\|x_{i}(k+1)-\gamma_{i j}\right\|^{2} \phi\left(\gamma_{i j}\right) n^{\top}\left(\gamma_{i j}\right) \frac{\partial \gamma_{i j}}{\partial x_{i}} d \gamma_{i j}$
Additionally $\sum_{i=1}^{N} \int \theta_{i} d u_{i}(k)=\sum_{i=1}^{N} \int \frac{\theta_{i}}{T_{s}} d x_{i}(k+1)$ with

$$
\begin{aligned}
& \sum_{i=1}^{N} \int \frac{\theta_{i}}{T_{s}} d x_{i}(k+1)=\sum_{i=1}^{N} \frac{p_{i}}{T_{s}} \int\left(\sum_{j \in \mathcal{N}_{i}} \int_{\mathbb{V}_{i}^{+} \cap \mathbb{V}_{j}^{+}}\right. \\
& \left.\left\|x_{i}(k+1)-\gamma_{i j}\right\|^{2} \phi\left(\gamma_{i j}\right) n^{\top}\left(\gamma_{i j}\right) \frac{\partial \gamma_{i j}}{\partial x_{i}} d \gamma_{i j}\right) d x_{i}(k+1)
\end{aligned}
$$

Since

$$
\left\|x_{i}-\gamma_{i j}\right\|=\left\|x_{j}-\gamma_{j i}\right\|, \frac{\partial \gamma_{i j}}{\partial x_{i}}=\frac{\partial \gamma_{j i}}{\partial x_{j}}, n\left(\gamma_{i j}\right)=-n\left(\gamma_{j i}\right)
$$

it is possible to write ${ }^{4}$

$$
\begin{aligned}
& \int_{\mathbb{V}_{i} \cap \mathbb{V}_{j}}\left\|x_{i}-\gamma_{i j}\right\|^{2} \phi\left(\gamma_{i j}\right) n^{\top}\left(\gamma_{i j}\right) \frac{\partial \gamma_{i j}}{\partial x_{i}} d \gamma_{i j} \\
& =-\int_{\mathbb{V}_{j} \cap \mathbb{V}_{i}}\left\|x_{j}-\gamma_{j i}\right\|^{2} \phi\left(\gamma_{j i}\right) n^{\top}\left(\gamma_{j i}\right) \frac{\partial \gamma_{j i}}{\partial x_{j}} d \gamma_{j i}
\end{aligned}
$$

and thus the integral

$\sum_{i=1}^{N} p \int\left(\sum_{j \in \mathcal{N}_{i}} \int_{\mathbb{V}_{i} \cap \mathbb{V}_{j}}\left\|x_{i}-\gamma_{i j}\right\|^{2} \phi\left(\gamma_{i j}\right) n^{\top}\left(\gamma_{i j}\right) \frac{\partial \gamma_{i j}}{\partial x_{i}} d \gamma_{i j}\right) d x_{i}$ vanishes. This result leads to $\sum_{i=1}^{N} \int \theta_{i} d u_{i}(k)=0$ and thus, the equation (23) becomes $H(k)=0$, proving that the control (21) can be considered as a discrete-time approximation of the optimal control solution of the problem (13).

\section{EQUIVALENCE BETWEEN DISCRETE-TIME APPROXIMATION OF CDOC AND CDOC-STABILITY PROOF}

The previous section presents the proposed discrete-time approximation of CDOC (14). It will be proved to converge to the CDOC of [10].

Theorem 2. If the sampling time $T_{s}$ goes to zero, then the next three statements are true:

$i$. The discretized equation (2) approaches the continuoustime dynamics (1);

ii. The discrete-time approximation of CDOC (14) approaches $C D O C$ (12);

iii. The HJB equation $\min _{u} H=0$ is ensured.

Proof. $i$. The first statement is obvious.

ii. Consider the discrete-time control solution (14). If $T_{s} \rightarrow$ 0 , this solution (14) approaches the limit value $u_{i}(t)=$ $-\frac{p}{r_{i}} \int_{\mathbb{V}_{i}}\left(x_{i}(t)-q\right) \phi(q) d q=-\sqrt{\frac{s_{i}}{r_{i}}} \int_{\mathbb{V}_{i}}\left(x_{i}(t)-q\right) \phi(q) d q$ which is exactly the CDOC solution, for $p^{2}=s_{i} r_{i}$ (see (6) and (11)).

${ }^{4}$ From the point of view of mass conservation, we consider the case when $\mathcal{X}=\mathbb{V}_{i} \cup \mathbb{V}_{j}$. If one part of the mass belonging to the $\mathbb{V}_{i}$ cell passes through the facet $\mathbb{V}_{i} \cap \mathbb{V}_{j}$ into the $\mathbb{V}_{j}$ cell, then $\mathbb{V}_{i}$ loses it but the cell $\mathbb{V}_{j}$ gets it. In general, the mass over $\mathcal{X}$ is conserved. 
iii. According to the definition of the total derivative of a multivariable function, it is inferred that

$$
\Delta V(k)=\sum_{i=1}^{N} \int \frac{\partial \Delta V(k)}{\partial u_{i}(k)} d u_{i}(k)
$$

Replacing $\Delta V(k)$ in the Hamiltonian (16) leads to $H(k)=\sum_{i=1}^{N}\left(s_{i}\left\|\int_{\mathbb{V}_{i}}\left(x_{i}(k)-q\right) \phi(q) d q\right\|^{2}+r_{i}\left\|u_{i}(k)\right\|^{2}\right)+$ $\frac{1}{T_{s}} \sum_{i=1}^{N} \int \frac{\partial \Delta V(k)}{\partial u_{i}(k)} d u_{i}(k)$. Substitute $\frac{\partial \Delta V(k)}{\partial u_{i}(k)}$ with and use (22) to obtain

$$
\begin{aligned}
& H(k)=\sum_{i=1}^{N}\left(s_{i}\left\|\int_{\mathbb{V}_{i}}\left(x_{i}(k)-q\right) \phi(q) d q\right\|^{2}+r_{i}\left\|u_{i}(k)\right\|^{2}\right) \\
& +\sum_{i=1}^{N} 2 p \int\left(M_{\mathbb{V}_{i}^{+}} x_{i}(k+1)-M_{\mathbb{V}_{i}^{+}} C M_{\mathbb{V}_{i}^{+}}\right)^{\top} d u_{i}(k)
\end{aligned}
$$

and thus

$$
\begin{aligned}
& H(k)=\sum_{i=1}^{N}\left(s_{i}\left\|\int_{\mathbb{V}_{i}}\left(x_{i}(k)-q\right) \phi(q) d q\right\|^{2}+r_{i}\left\|u_{i}(k)\right\|^{2}\right) \\
& +\sum_{i=1}^{N} 2 p M_{\mathbb{V}_{i}^{+}}\left(\int x_{i}^{\top}(k+1) d u_{i}(k)-C M_{\mathbb{V}_{i}^{+}}^{\top} \int d u_{i}(k)\right)
\end{aligned}
$$

Using the dynamics (2), this can be rewritten as

$$
\begin{aligned}
& H(k)=\sum_{i=1}^{N}\left(s_{i}\left\|\int_{\mathbb{V}_{i}}\left(x_{i}(k)-q\right) \phi(q) d q\right\|^{2}+r_{i}\left\|u_{i}(k)\right\|^{2}\right) \\
& +\sum_{i=1}^{N} 2 p M_{\mathbb{V}_{i}^{+}}\left(T_{s} \frac{\left\|u_{i}(k)\right\|^{2}}{2}+\left(x_{i}(k)-C M_{\mathbb{V}_{i}^{+}}\right)^{\top} u_{i}(k)\right)
\end{aligned}
$$

Regrouping the terms in $\left\|u_{i}(k)\right\|^{2}$, the expression (35) becomes $H(k)=\sum_{i=1}^{N}\left(s_{i}\left\|\int_{\mathbb{V}_{i}}\left(x_{i}(k)-q\right) \phi(q) d q\right\|^{2}+\right.$ $\left.\left(r_{i}+p T_{s} M_{\mathbb{V}_{i}^{+}}\right)\left\|u_{i}(k)\right\|^{2}+2 p M_{\mathbb{V}_{i}^{+}}\left(x_{i}(k)-C M_{\mathbb{V}_{i}^{+}}\right)^{\top} u_{i}(k)\right)$. Replacing $u_{i}(k)$ by the solution (14), this is equivalent to $H(k)=\sum_{i=1}^{N}\left(s_{i}\left\|\int_{\mathbb{V}_{i}}\left(x_{i}(k)-q\right) \phi(q) d q\right\|^{2}-\right.$ $\left.\frac{p^{2}}{r_{i}+p T_{s} M_{\mathbb{V}_{i}^{+}}}\left\|\int_{\mathbb{V}_{i}^{+}}\left(x_{i}(k)-q\right) \phi(q) d q\right\|^{2}\right)$.

When $T_{s} \rightarrow 0$, using $C M_{\mathbb{V}_{i}^{+}} \rightarrow C M_{\mathbb{V}_{i}}$ and $M_{\mathbb{V}_{i}^{+}} \rightarrow M_{\mathbb{V}_{i}}$, the control law (14) becomes

$$
u_{i}(t)=-\frac{p}{r_{i}} \int_{\mathbb{V}_{i}}\left(x_{i}(t)-q\right) \phi(q) d q
$$

which is further used to find a simplified form of $\min H$

$$
\min _{u} H=\sum_{i=1}^{N}\left(s_{i}-\frac{p^{2}}{r_{i}}\right)\left\|\int_{\mathbb{V}_{i}}\left(x_{i}(k)-q\right) \phi(q) d q\right\|^{2}
$$

Using $p^{2}=s_{i} r_{i}$, we conclude that if $T_{s} \rightarrow 0$ then $\min _{u} H \rightarrow 0$. The proof is thus completed.

\section{NUMERICAL EXAMPLE}

We consider a Multi-Agent system $\boldsymbol{\Sigma}$ composed of $N=$ 7 homogeneous agents having the common dynamics (2), with the sampling time $T_{s}=0.01 \mathrm{~s}, x_{i} \in \mathbb{R}^{2}$ and $u_{i} \in \mathbb{R}^{2}$ denoting respectively the agents position and speed.

The agents are deployed within a bounded region $\mathcal{X}=$ $\operatorname{conv}\{(0,0),(0,6),(6,6),(6,0)\}$, see Fig. 1. The density function over $\mathcal{X}$ is uniform, i.e. $\phi(q)=1, \forall q \in \mathcal{X}$.

In Figs. 1 and 3 , the blue lines represent the motion of the agents in the considered discrete-time case. The agents initial positions $x_{i}(0)$ are marked by the red points (see the zoom shown in Figs. 1 and 3). The last configuration of the entire MAS is shown in each figure, with the green dots denoting the last positions of the agents. The evolution of the coverage criterion $M_{\mathbb{V}_{i}}\left\|x_{i}-C M_{\mathbb{V}_{i}}\right\|$ for each agent is shown in Figs. 2 and 4.

Two scenarios are considered. In the first scenario, we apply the decentralized control (14) with the weighting coefficients $s_{i}=10$ and $r_{i}=1$. The deployment result is shown in Fig. 1 with the evolution of the agents and also the Voronoi partition obtained after 100 sampling periods. The coverage criterion curves in Fig. 2 drop asymptotically to zero, proving that the entire MAS $\boldsymbol{\Sigma}$ is close to a CVC.

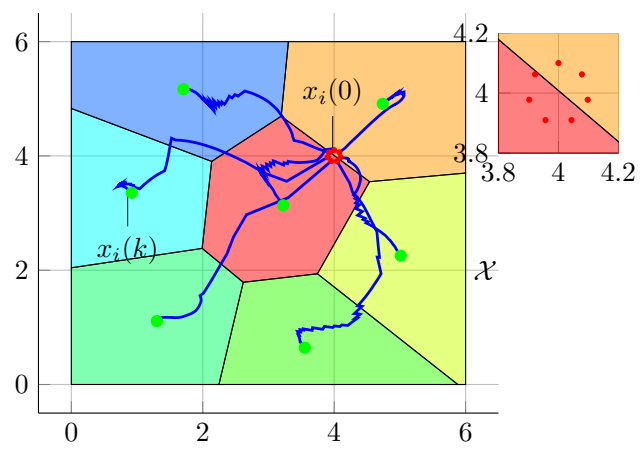

Fig. 1. Coverage of $\mathcal{X}$ with $s_{i}=10$ and $r_{i}=1$.

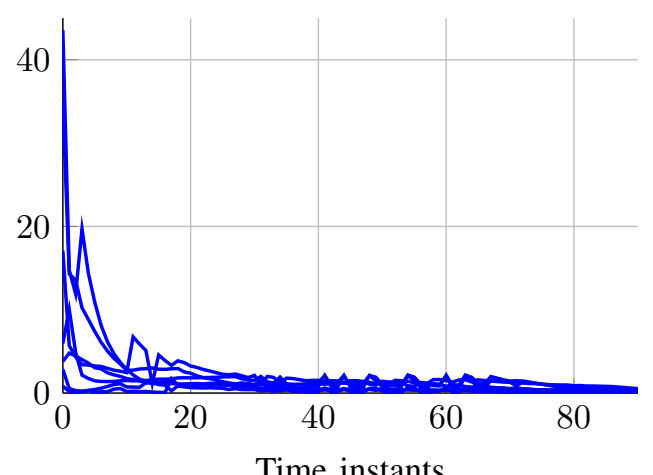

Fig. 2. Coverage criterion of $N$ agents with $s_{i}=10$ and $r_{i}=1$.

In the second scenario, the weighting coefficients are $s_{i}=$ 100 and $r_{i}=1$. The MAS converges into a CVC different from the previous scenario (notice that a CVC is not not unique [17]) with a faster convergence rate. The drop to zero 
of the coverage criterion proves that $\boldsymbol{\Sigma}$ approaches the CVC (see Fig 2), although there are some discontinuities due to the abrupt change of the shape of the dynamic Voronoi cells.

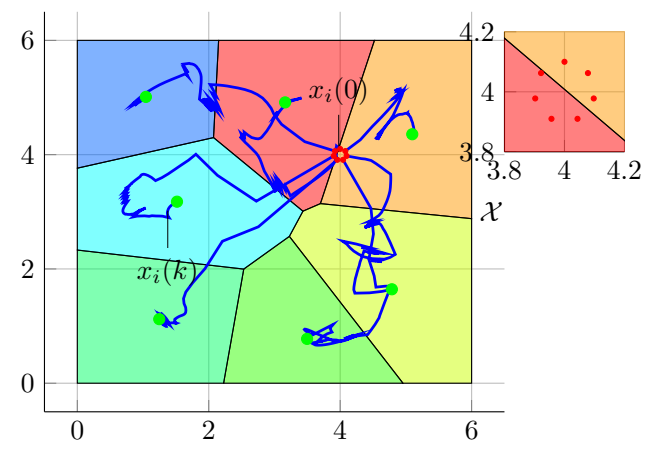

Fig. 3. Coverage of $\mathcal{X}$ with $s_{i}=100$ and $r_{i}=1$.

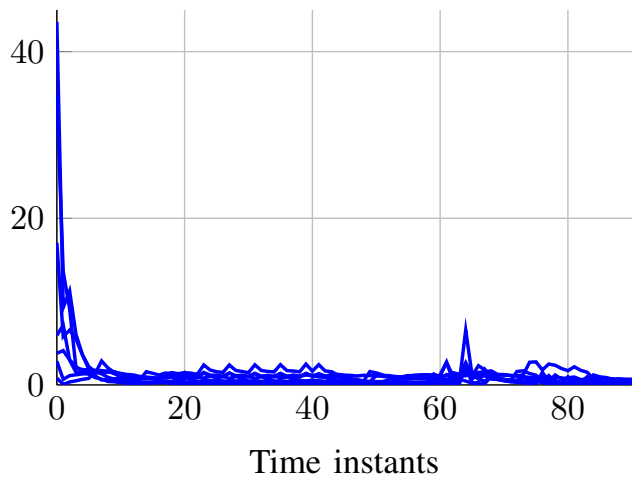

Fig. 4. Coverage criterion of $N$ agents with $s_{i}=100$ and $r_{i}=1$.

\section{CONCLUSION}

This paper provides a novel discrete-time decentralized control for the Multi-Agent Voronoi-based coverage/deployment problem, by following the optimal control framework. Similar to the continuous-time case, the discretetime solution is also spatially distributed over Delaunay graphs. One of the contributions consists of taking into account the prediction of the Voronoi partition. However, finding an explicit equation to predict this partition appears to be complicated and it is part of our current research work. Some simulations showed small oscillations/discontinuities in the evolution of the centroids which will be analyzed in the future. Another interesting topic to be addressed in future work relates to the choice of the weighting coefficients for the energy-efficiency problem. Extending the control solutions for the case of finite horizon is part of current work. Future work will focus on the application of the proposed results on Smart Grid, related to distributed/decentralized energy production and distribution.

\section{ACKNOWLEDGMENT}

The first author gratefully acknowledges the support of the LIA Project between L2S and SYTACom. The second author would like to acknowledge the Natural Sciences and Engineering Research Council of Canada (NSERC) for funding his contribution to this research.

\section{REFERENCES}

[1] R. M. Murray, "Recent research in cooperative control of multivehicle systems," Journal of Dynamic Systems, Measurement, and Control, vol. 129, no. 5, pp. 571-583, 2007.

[2] M. Schwager, J.-J. E. Slotine, and D. Rus, "Unifying geometric, probabilistic, and potential field approaches to multi-robot coverage control," in ISRR. Springer, 2009, pp. 21-38.

[3] Y. Song, B. Wang, Z. Shi, K. R. Pattipati, and S. Gupta, "Distributed algorithms for energy-efficient even self-deployment in mobile sensor networks," Mobile Computing, IEEE Transactions on, vol. 13, no. 5, pp. 1035-1047, 2014.

[4] E. Bakolas and P. Tsiotras, "Optimal partitioning for spatiotemporal coverage in a drift field," Automatica, vol. 49, no. 7, pp. 2064-2073, 2013.

[5] K. Sugihara, "Voronoi diagrams in a river," International Journal of Computational Geometry \& Applications, vol. 2, no. 01, pp. 29-48, 1992.

[6] R. O. Saber and R. M. Murray, "Flocking with obstacle avoidance: cooperation with limited communication in mobile networks," in Decision and Control, 2003. Proceedings. 42nd IEEE Conference on, vol. 2. IEEE, 2003, pp. 2022-2028.

[7] H. G. Tanner, "Flocking with obstacle avoidance in switching networks of interconnected vehicles," in ICRA, vol. 3. IEEE, 2004, pp. 30063011.

[8] G. Voronoï, "Nouvelles applications des paramètres continus à la théorie des formes quadratiques. deuxième mémoire. Recherches sur les parallélloèdres primitifs." Journal für die reine und angewandte Mathematik, vol. 134, pp. 198-287, 1908.

[9] A. Kwok and S. Martinez, "Deployment algorithms for a powerconstrained mobile sensor network," International Journal of Robust and Nonlinear Control, vol. 20, no. 7, pp. 745-763, 2010.

[10] M. Moarref and L. Rodrigues, "An optimal control approach to decentralized energy-efficient coverage problems," in IFAC World Congress, Cape Town, South Africa, 2014

[11] J. Cortes, S. Martinez, T. Karatas, and F. Bullo, "Coverage control for mobile sensing networks," in Robotics and Automation, 2002. ICRA'02, vol. 2, 2002, pp. 1327-1332.

[12] S. P. Lloyd, "Least squares quantization in PCM," IEEE Transactions on Information Theory, vol. 28, no. 2, pp. 129-137, 1982.

[13] Y. Yan and Y. Mostofi, "Utilizing mobility to minimize the total communication and motion energy consumption of a robotic operation," in Workshop on Distributed Estimation and Control in Networked Systems, 2012, pp. 180-185.

[14] J. Cortes, S. Martinez, and F. Bullo, "Spatially-distributed coverage optimization and control with limited-range interactions," ESAIM: Control, Optimisation and Calculus of Variations, vol. 11, no. 04, pp. 691-719, 2005.

[15] H. K. Khalil and J. Grizzle, Nonlinear systems. Prentice Hall, New Jersey, 1996, vol. 3.

[16] R. Bellman, "Dynamic programming and a new formalism in the calculus of variations," Proceedings of the national academy of sciences, vol. 40, no. 4, pp. 231-235, 1954.

[17] Q. Du, V. Faber, and M. Gunzburger, "Centroidal Voronoi tessellations: applications and algorithms," SIAM review, vol. 41, no. 4, pp. 637676, 1999. 